\title{
Er:YAG Laser as an Advanced Periodontal Surgical Technique
}

\author{
Amita Sharma ${ }^{1}$, Parveen Ranga ${ }^{1}$ and Sonam Sharma ${ }^{2 *}$ \\ ${ }^{1}$ Department of Dentistry, Shaheed Hasan Khan Mewati Government Medical College, Nalhar, Nuh, Haryana, India \\ ${ }^{2}$ Department of Pathology, Kalpana Chawla Government Medical College, Karnal, Haryana, India
}

\begin{abstract}
Lasers were introduced into the field of dentistry as they performed work more precisely and effectively with less discomfort to the patient by minimizing pain and recovery time. Every discipline of dentistry has been positively affected with the use of laser technology including Oral Medicine, Oral surgery, Pediatric and Operative Dentistry, Periodontics and Oral Implantology, Prosthetic Dentistry. Treatment with lasers provides a hope of overcoming the disadvantages of conventional dental procedures. In this paper, a brief history of the mechanism and uses of the Erbium-doped:Yttrium-Aluminium-Garnet (Er:YAG) laser is presented, which is the most commonly used tool in Periodontics and Oral Implantology.
\end{abstract}

Keywords: Lasers, Er:YAG, Periodontics

\section{Introduction}

Laser is an acronym for "light amplification by stimulated emission of radiation." The stimulated emission of a photon by an excited atom, which triggers the release of a subsequent photon, is responsible for generation of a coherent, monochromatic, and collimated form of light, or laser. ${ }^{[1]}$ Laser can concentrate light energy and exert a strong effect, by targeting tissue at an energy level much lower than natural light. The wavelength of a laser determines its characteristics. Evidence suggests its use in initial periodontal therapy, surgery, and more recently, its utility in salvaging implant opens up a wide range of applications. ${ }^{[2]}$

Nowadays numerous laser systems are available for dental use (Table 1). Neodymium-doped:YttriumAluminium-Garnet (Nd:YAG), carbon dioxide $\left(\mathrm{CO}_{2}\right)$ and semiconductor diode lasers have already been approved by the United States Food and Drug Administration for soft tissue treatment in oral cavity. The Erbium-doped: Yttrium-Aluminium-Garnet (Er:YAG) laser was approved in 1997 for hard tissue treatment in dentistry and recent studies and developments reported many positive results.
This suggests that the Er:YAG laser system is a promising apparatus, which will be able to revolutionize and improve clinical dental practice, in particular periodontal treatment. [3]

\section{History of lasers}

Maiman invented the first laser device in May 16, 1960 based on theories derived by Einstein in the early 1900 at Hughes Research Laboratories in Malibu, California, by using a cylinder of synthetic ruby measuring $1 \mathrm{~cm}$ in diameter and $2 \mathrm{~cm}$ long, with the silver-coated ends to make them reflective and able to serve as a Fabry-Perot resonator. He used photographic flash lamps as the lasers pump source. This initial breakthrough in the modern optics heralded the birth of laser science and the following decade witnessed a spectacular period of growth with the emergence of new and novel laser systems. Just a year after the ruby laser, the first gas laser i.e. helium-neon ( $\mathrm{HeNe})$ laser was introduced by Javan et al in $1961 .{ }^{[4]}$ This was soon followed by other systems using gases like $\mathrm{CO}_{2}$, Excimers or excited dimmers (XeF, $\mathrm{KrF}, \mathrm{ArF}),{ }^{[5]}$ Copper vapours, ${ }^{[6]}$ Argon ion ${ }^{[7]}$ and Krypton ion. New solid-state laser were also introduced showing new optical crystals as an active

Table 1: Different lasers used in dentistry with their wavelength targeting the tissue ${ }^{[3]}$.

\begin{tabular}{|c|c|c|c|}
\hline Type of lasers & Wavelength $(\mathbf{n m})$ pulse mode & Chromophores used & Target tissue \\
\hline Diode & $850-1064$ & Pigments Hemoglobin, Melanin & Gingiva, mucosa \\
\hline Nd:YAG & 1064 & Pigments Hemoglobin, Melanin & Gingiva, mucosa \\
\hline Er:YAG & 2940 & Water, Hydroxyapatite & Gingiva, mucosa, enamel, dentin, bone \\
\hline Er, Cr:YSGG & 2860 & Water, Hydroxyapatite & Gingiva, mucosa, enamel, dentin, bone \\
\hline CO2 & 10640 & Mucosa & Water , Gingiva \\
\hline
\end{tabular}


medium. The most useful among these was Nd:YAG and Er:YAG lasers. ${ }^{[8]}$

\section{Physics behind lasers}

In ordinary light, the photons (energy) travel in different wavelengths in all directions, whereas the unique characteristics of the laser light are that it is monochromatic, coherent, and collimated. Laser light has a single wavelength, and all the light waves are in phase and in a precise beam in one direction, instead of spreading in space. In the visible portion of the electromagnetic spectrum, the single wavelength also means a single color. These properties allow the concentration of a tremendous amount of energy to a small area. Depending on the wavelength and the properties of the target tissue or material, the energy is absorbed, reflected, scattered, or allowed to go through. The interaction plus time and power determine the results. ${ }^{[1]}$

\section{Mechanism of action}

Er:YAG wavelengths is $2,940 \mathrm{~nm}$ which interact with both soft tissue chromophore and hard tissue. These wavelengths have an affinity for (carbonated) hydroxyapatite and water chromophores. However, although the water content of enamel and dentine is very low (3-5\% in enamel and $13-$ $15 \%$ in dentine), it is the configuration of the lasers emission modes that defines the underlying nature of tissue ablation. These inherent absorption qualities allow erbium lasers to ablate tooth and bone. During Er:YAG laser irradiation, the laser energy is absorbed selectively by water molecules and hydrous organic components of biological tissues, causing evaporation of water and organic components and resulting in thermal effects due to the heat generated by this process - 'photothermal evaporation'.

In hard tissue procedures, the water vapour production induces an increase of internal pressure within the tissue, resulting in explosive expansion called 'micro explosion.' ${ }^{[9,10]}$ These dynamic effects cause mechanical tissue collapse, resulting in a 'thermo-mechanical' or 'photo-mechanical' ablation. ${ }^{[11]}$ This phenomenon has also been referred to as 'water mediated explosive ablation' [12,13] . Erbium lasers are unique as they are the only lasers that can cut both hard and soft tissues with minimal heat-related side effects. ${ }^{[14]}$

\section{Use in periodontology}

The erbium wavelengths present the broadest range of application for clinical dentistry and are likely the most suitable lasers for periodontal therapy. The erbium lasers are effective in removing calculus and reducing pocket probing depth (PPD). Dental calculus contains water in its structural micro-pores and in its intrinsic components. Since the Er:YAG laser has the ability to ablate dental hard tissues, it was expected to be capable of removing dental calculus at much lower energy levels..$^{[15,16]}$

\section{A. Scaling and currettage}

Er:YAG laser is contraindicated in Supra-gingival scaling on enamel surface as complete calculus removal without affecting the underlying enamel is difficult. However, it is clinical proven that this laser in subgingival scaling, not only removes the calculus but also help in removal of contaminated cementum which gives promising result in periodontal pocket treatment and root surface debridement, including root preparation and pocket curettage. ${ }^{[17]}$

\section{B. Laser-assisted in new attachment procedure}

Er:YAG laser has the potential to remove toxins diffused into the root cementum and has a bactericidal effect and is also effective in removing the absorbed root surface endotoxins. The haemostatic effect is weaker than any other lasers, but the healing of the laser wound is relatively fast and comparable to that of a scalpel wound. Laser irradiation has been reported to exhibit bactericidal and detoxification effects without producing a smear layer and the laser treated root surface might therefore provide favourable conditions for the attachment of periodontal tissue. ${ }^{[18]}$

\section{Effects of Er:YAG laser on Periodontal Therapy}

1. It decreases the nerve sensitivity and significantly reduces the perception of pain by decreasing the transmission of pain signal from injured parts of body to the brain.

2. Its application increases the production and release of endorphins and enkephalins which are natural pain-relieving chemicals within our bodies.

3. Laser therapy helps in reduction of inflammation causing the smaller arteries and lymph vessels of the body to increase in size by mechanism called vasodilatation.

4. It promotes lymphatic drainage which also aids in this vital healing process.

5. Photons of light emitted by therapeutic lasers penetrate deeply into the tissues of the body to stimulate the production centers of individual cells which accelerate the tissue repair and cell growth. This stimulation increases the energy available to these cells, causing them to absorb nutrients and expel waste products more rapidly. ${ }^{[19]}$ 
D. Laser works as a Substitute to Antibiotics Used In Periodontitits

Periodontal disease is a chronic inflammatory disease caused by a bacterial infection. Hence the bactericidal and detoxifying effect of laser treatment is advantageous in periodontal therapy. ${ }^{[20]}$ The Er:YAG laser shows the bactericidal effect against bacterias like Porphyromonas gingivalis and Aggregatibacter actinomycetemcomitans, hence provides a nonantibiotic solution which ultimately decreases the antibiotic resistance. ${ }^{[21]}$

\section{E. Laser \& Implants}

Gingival enlargement is relatively common around implants when they are loaded with removable prosthesis. Lasers can be used for the hyperplasia removal as well as in the treatment for peri-implantitis. Significant improvement in the treatment of periimplantitis also occurs with the addition of laser therapy. ${ }^{[22]}$ Due to its high bactericidal effect without heat generation around implants, it can be used in the maintenance of implants. ${ }^{[2]}$

\section{Advantages of using lasers in the periodontal therapy} include ${ }^{[23]}$ :

1. Less pain

2. Less need for anesthetics (an advantage for medically compromised patients)

3. No risk of bacteremia

4. Excellent wound healing; no scar tissue formation

5. Bleeding control (dependent on the wavelength and power settings);

6. Usually no need for sutures

7. Use of fewer instruments and materials and no need for autoclaving (economic advantages)

8. Ability to remove both hard and soft tissues

9. Lasers can be used in combination with scalpels (however, the laser is a tool and not a panacea).

Disadvantages of using lasers in periodontal therapy include ${ }^{[23]}$ :

1. Relatively high cost of the devices

2. A need for additional education (especially in basic physics)

3. Every wavelength has different properties

4. The need for implementation of safety measures (i.e. goggle use)

\section{Laser Safety}

1. General safety include laser warning sign outside the clinic
2. Use of barriers within the operatory side

3. Use of eyewear to protect against reflected laser light or accidental direct exposure.

4. High volume suction must be used to evacuate the plume from tissue ablation.

5. Several authors have studied the thermal effect of lasers on the periodontal ligament and surrounding bone. Hence, periodontal tissues are not damaged if the temperature increase is kept below $5^{\circ} \mathrm{C}$.

6. A threshold temperature increase of $7^{\circ} \mathrm{C}$ is commonly considered as the highest thermal change, which is biologically acceptable to avoid periodontal damage. [24]

\section{Conclusion}

Er:YAG laser possess characteristics suitable for dental treatment, due to its dual ability to ablate soft and hard tissues with minimal damage. ${ }^{[25]}$ In addition, its bactericidal effect with elimination of Lipopolysaccharide, ability to remove bacterial plaque and calculus, irradiation effect limited to an ultra-thin layer of tissue, faster bone and soft tissue repair, makes it a promising tool for periodontal treatment including scaling and root surface debridement. Finally, in order to have a successful long term periodontal treatment, patients need to be motivated. It is not so much the technology but the motivation and psychology that matter when it comes to practice of oral hygiene before, during and after the periodontal treatment to maintain a good and stable periodontal condition. ${ }^{[26]}$

\section{References:}

1. Aoki A, Sasaki KM, Watanabe H, Ishikawa I. Lasers in nonsurgical periodontal therapy. Periodontol 2000. 2004;36:59-97.

2. Sagar K, Kaur A, Patel P, Kumar V, Narang S and Ranga P. Diode laser as an established tool in periodontics-a review. Am J Oral Med Radiol. 2015;2(2):54-60.

3. Lee DH. Application of Laser in Periodontics. A New Approach in Periodontal treatment. Dental Bulletin, Hong Kong Medical Diary. 2007;12(10):23-27.

4. Javan A, Bennett WR, Harriot DR. Population Inversion and Continuous Optical Maser Oscillation in a Gas Discharge Containing a He-Ne Mixture. Phys Rev Lett. 1961;6:106-10.

5. Patel CKN. Continuous-Wave Laser Action on Vibrational-Rotational Transitions of CO2. Phys Rev A.1964;136:1187-93.

6. Burnham R, Djeu N. Ultraviolet-preionised discharge pumped lasers in $\mathrm{XeF}, \mathrm{KrF}$ and ArF. Appl Phys Lett 1964;4:128-30.

7. Walter WT, Piltch M, Solimene N, Gould G. Pulsedlaser action in atomic copper vapour. Bull Am Phys Soc. 1966;11:113. 
8. Bridges WB. Laser oscillation in singly ionised argon in the visible spectrum. Appl Phys Lett. 1964;4:128-30.

9. Hibst R, Keller U. Experimental studies of the application of the Er:YAG laser on dental hard substances. I. Measurement of the ablation rate. Lasers Surg Med.1989;9(4):338-44.

10. Keller U, Hibst R. Experimental studies of the application of the Er:YAG laser on dental hard substances. II. Light microscopic and SEM investigations. Lasers Surg Med. 1989; 9(4):345-51.

11. Koort HJ, Frentzen M. Laser effects on dental hard tissue In: Miserendino LJ, Pick RM, editors. Lasers in Dentistry Chicago: Quintessence, 1995; pp 57-70.

12. Fried D. IR laser ablation of dental enamel. Proc. SPIE 2000; 3910:136-148.

13. Seka W, Featherstone JD, Fried D, Visuri SR, Walsh JT. Laser ablation of dental hard tissue: from explosive ablation to plasma-mediated ablation. Proc. SPIE, 1996;2672:144-58.

14. Cobb CM. Lasers in periodontics: a review of the literature. J Periodontol. 2006;77(4):545-64.

15. Aoki A, Miura M, Akiyama F, Nakagawa N, Tanaka J, Oda S, Watanabe H, Ishikawa I. In vitro evaluation of Er:YAG laser scaling of subgingival calculus in comparison with ultrasonic scaling. J Periodontal Res. 2000;35:266-77.

16. Frentzen M, Braun A, Aniol D. Er:YAG laser scaling of diseased root surfaces. J Periodontol. 2002;73:524-30.

17. Minovska A. Nd:YAG and Er:YAG Wavelengths Used as Therapeutic Tool in Periodontal Disease. Balk J Stom. 2011;15:59-65.
18. Yamaguchi H, Kobayashi K, Osada R, Sakuraba E, Nomura $\mathrm{T}$, Arai T, et al. Effects of irradiation of an erbium: YAG laser on root surfaces. J Periodontol. 1997;68(12):1151-5.

19. Schwarz F, Jepsen S, Herten M, Aoki A, Sculean A, Becker J.Immunohistochemical characterization of periodontal wound healing following non-surgical treatment with fluorescence controlled Er:YAG laser radiation in dogs. Lasers Surg Med. 2007;39(5):428-40.

20. Haraszthy, Zambon, Ciancio, et al. Microbiological effects of $810 \mathrm{~nm}$ diode laser treatment of periodontal pockets. AADR 2006, abs 1163.

21. Ando Y, Watanabe H, Ishikawa I. Bactericidal effect of erbium:YAG laser on periodontopathic bacteria. Lasers Surg Med. 1996;19(2):190-200.

22. Bach G, Neckel C, Mall C, Krekeler G. Conventional versus laser assisted therapy of peri-implantitis: a five-year comparative study. Implant Dent. 2000; 9:247-51.

23. Khan MS. Laser - A Paradigm Approach in Periodontal Surgery - A Review Article. IOSR JDMS. 2017;16(9):31-4.

24. Piccione PJ. Dental laser safety. Dent Clin North Am. 2004;48:795-807.

25. Melcer J. Latest treatment in dentistry by means of the $\mathrm{CO} 2$ laser beam. Lasers Surg Med. 1986;6:396-8.

26. Asnaashari M, Moeini M. Effectiveness of Lasers in the Treatment of Dentin Hypersensitivity. J Lasers Med Sci $2013 ; 4(1): 1-7$.

*Corresponding author:

Dr. Sonam Sharma, Assistant Professor, Department of Pathology, Kalpana Chawla Government Medical College, Karnal -132001, Haryana, India Phone: +91 09999841393

Email: drsonamsharma@gmail.com

Financial or other Competing Interests: None. 